\title{
Continuous Trait-Based Particle Swarm Optimisation (CTB-PSO)
}

\author{
Ed Keedwell, Mark Morley, and Darren Croft \\ University of Exeter, Exeter, EX4 4QF UK \\ \{E.C.Keedwell, M.S.Morley, D.P.Croft\}@exeter.ac.uk
}

\begin{abstract}
Previous work on swarms with variation in particle behaviours has focussed on discrete behaviours. In this paper we investigate a traitbased approach where particles in the swarm display degrees of different behaviour. Results show that trait-based diversity in the swarm leads to better performance than standard PSO and discrete behaviour variation.
\end{abstract}

Keywords: heterogeneous particle swarm optimisation, behavioural variation, behavioural traits.

\section{Introduction}

In natural flocks, individuals are often of the same species, but there exists considerable variation in the traits possessed by each individual. In much the same way as humans display varied levels of aggression, gregariousness and inquisitiveness, so do the animals on which PSO is based [1]. Recent research has shown that this disparity of behaviour is very important in the ability of the flock to solve problems effectively, which might have profound implications for PSO. One of the key aspects is that although certain behaviour types (e.g. more adventurous individuals) might individually be better at problem solving; selecting for a group that all have adventurous traits has been shown to reduce the performance of the flock as a whole [1]. Therefore a flock that has a variety of behaviours leads to better performance in natural and it is this that motivates the work here. This paper explores a variant of PSO known as Continuous TraitBased PSO (CTB-PSO) where individuals within a swarm have traits based on a continuous scale as opposed to discrete behaviour groupings.

There is a considerable body of work that explores behaviour variation through the use of multiple species, but the most relevant single-species work is that of Andries Engelbrecht [2] and the Heterogenous PSO algorithm(HPSO). HPSO is similar in motivation to CTB-PSO but exploits intra-species variation via a discrete 'behaviour' pool. CTB-PSO differs from HPSO as it achieves heterogeneity through the generation of $c 1$ and $c 2$ coefficient values from a Gaussian distribution with known mean and standard deviation to determine different behaviours for each of the particles in the initialisation stage, in line with what is known about behavioural traits in animals. Once determined, these coefficient selections remain constant for each particle for the duration of the optimisation and the remainder of the PSO algorithm is then run in a standard fashion. 


\section{Method}

CTB modifies standard PSO by varying the extent to which each particle is influenced by the global and personal best positions within the group.

1. Generate a random population of particles.

2. For each particle, randomly select $c 1$ and $c 2$ coefficient values drawn from a Gaussian distribution with a specified mean and standard deviation.

3. Execute remainder of PSO as normal.

\section{Experimental Setup and Results}

The algorithm was run for 1000 generations with a swarm size of 50, and for 30 repeated trials to account for the effect of the random seed. CTB-PSO was tested on four test functions taken from the literature: Absolute Value, Spherical, Griewank and Ackley and results for 2 functions are shown below. For further information on the functions see [2]. Experiments were conducted on problem sizes from 10 to 100 dimensions. The mean and standard deviation of the Gaussian applied to $c 1$ and $c 2$ was modified, with best performance observed with mean $=2.0$ and stddev $=0.5$. The standard PSO operates with fixed $c 1$ and $c 2$ coefficients of 2.0 and HPSO results are taken from [2].

Table 1. Comparison with HPSO on Two Test Functions From the Literature

\begin{tabular}{|l|l|l|l|r|}
\hline Dimensions & HPSO-Griewank & CTB-PSO-Griewank & HPSO-Ackley & CTB-PSO-Ackley \\
\hline 10 & $\mathbf{0 . 0 7 8 2}$ & 0.130 & $3.99 \mathrm{E}-15$ & $\mathbf{2 . 0 1 E - 1 8}$ \\
\hline 30 & 0.0407 & $\mathbf{0 . 0 0 8 5}$ & 1.20 & $\mathbf{0 . 2 7}$ \\
\hline 50 & 0.154 & $\mathbf{0 . 0 0 4 8}$ & 2.87 & $\mathbf{1 . 9 4}$ \\
\hline 100 & 3.61 & $\mathbf{0 . 0 2}$ & $\mathbf{2 . 8 7}$ & 3.58 \\
\hline
\end{tabular}

The results for all four functions (not shown) show that CTB-PSO improves on our standard PSO formulation at smaller dimensionalities. The comparison in Table 1 shows that in the majority of cases, CTB-PSO improves on the performance of HPSO in both functions indicating that trait-based PSO could offer improved performance over homogeneous and discrete-heterogeneous PSO.

Acknowledgement - This work was funded by the 'Bridging the Gaps: Exeter Science Exchange' project funded by the EPSRC (EP/I001433/1).

\section{References}

1. Croft, D. et. al. (2009) 'Behavioural trait assortment in a social network: patterns and implications' Behavioral Ecology and Sociobiology, vol. 63, pp. 1495-1503

2. A. Engelbrecht, (2010) 'Heterogeneous Particle Swarm Optimisation', in ANTS 2010, Brussels, 2010. 